\title{
PROGRESSIVE ENCEPHALOPATHY IN BOYS WITH SYMPTOMS OF RETT SYNDROME AND MECP2 MUTATIONS
}

Four young boys with neonatal onset of encephalopathy, a progressive course, and MECP2 mutations are reported from the University of Alabama, Birmingham, AL Symptoms suggestive of Rett syndrome included failure to thrive, respiratory insufficiency, microcephaly, hypotonia, movement disorder, with myoclonic, dyskinetic, and choreiform patterns, and repetitive face scratching or nose rubbing stereotypies. MECP2 mutations, characteristic of girls with Rett syndrome, are previously reported in 11 boys with progressive encephalopathy, 7 having affected sisters. The authors report 4 de novo male cases, 3 of whom died at 14 to 27 months of age. Details of the 11 previously published male patients are tabulated, 10 of whom died in infancy. (Kankirawatana P, Leonard H, Ellaway C et al. Early progressive encephalopathy in boys and MECP2 mutations. Neurology July ( 1 of 2) 2006;67:164-166). (Reprints: Dr Alan K Percy, CIRC 320E, $15303^{\text {rd }}$ Avenue South, Birmingham, AL 35294).

COMMENT. Young boys presenting with early progressive encephalopathy, respiratory insufficiency, movement disorder, seizures, or changes in muscle tone should be tested for $M E C P 2$ pathology and a possible diagnosis of Rett syndrome. Molecular confirmation is important in defining the cause and prognosis of this progressive encephalopathy and in providing genetic counseling.

NEUROMUSCULAR DISEASES

\section{INFANTILE ONSET MYASTHENIA GRAVIS WITH MUSK ANTIBODIES}

A late infantile case of myasthenia gravis (MG) with anti-muscle-specific receptor tyrosine kinase (MuSK) high antibody ( $\mathrm{Ab}$ ) titer is reported from Kyushu University, Fukuoka; and Nagasaki University, Japan. The onset with bilateral ptosis and abnormal phonation occurred after febrile seizures at 2 years of age. The diagnosis by a positive edrophonium test at age 3 years was followed by treatment with oral prednisolone. Ocular symptoms responded but bulbar symptoms persisted and worsened by age 12 years. The addition of pyridostigmine and increase in dose of steroid had limited benefit. Thymectomy was performed at age 26 years, with no response. On admission at age 29 , he had marked facial weakness, nasal voice and dysphagia, and choked on drinking water. The tongue was atrophic with a triple-furrowed appearance, and the neck and proximal muscles of extremities were weak, without fasciculations. Tendon reflexes and sensation were normal. Edrophonium infusion improved speech and swallowing. Anti-MuSK Ab measured by radioimmunoassay was $4.80 \mathrm{nmol} / \mathrm{L}$ (normal, $<0.05$ ). His voice, swallowing and muscle strength improved after plasma exchange using Spectra. (Murai H, Noda T, Himeno E et al. Infantile onset myasthenia gravis with MuSK antibodies. Neurology July (1 of 2) 2006;67:174-4). 
COMMENT. The authors cite reports of anti-MuSK MG mainly in middle-aged female adults, and a frequency of anti-MuSK Ab in AChR seronegative MG in $38 \%$ to $47 \%$ of cases. The present late infantile case suggests a wider spectrum of disease. The response to immunotherapies was thought to rule out a diagnosis of congenital MG.

A study of 77 juvenile myasthenia gravis patients, evaluated over a period of 34 years at the National Institute of Mental Health, Bangalore, India, and compared to 290 patients with MG onset after 15 years of age, showed $30 \%$ had ocular myasthenia and the remainder had generalized MG at onset (Ashraf VV et al. Acta Neurol Scand Aug 2006;114:119-123). MG was familial in $10(13 \%)$, with siblings affected. Treatment with corticosteroids was used in $52(67 \%)$, and 5 received azathioprine in addition. Thymectomy was performed in 11 , thymic histology showing hyperplasia in 8 and thymoma in 1 . In 64 patients with follow-up information (mean 6.2 years; range 6 mos to $25 \mathrm{yrs),} 10$ (18.5\%) had complete remission for at least one year without therapy, $25(39 \%)$ were asymptomatic, $28(44 \%)$ were partially improved, and $9(14 \%)$ where unchanged or worse. Two died, and 4 had crises.

\section{A NOVEL VARIANT OF AUTOSOMAL RECESSIVE LOWER MOTOR NEURON DISEASE}

A genome-wide linkage analysis to map the underlying genetic defect was performed in a consanguineous African family with five patients affected with a novel variant of autosomal recessive lower motor neuron disease (LMND), in a study at Hopital Necker Enfants Malades and other centers, Paris, France. In 4 of the 5 patients having a severe phenotype, symptoms appeared during infancy ( 2 to 3.5 years), with proximal muscle weakness predominating in the lower limbs and early involvement of foot and hand muscles. Paralysis spread to become generalized, except for the cranial nerves, and by age 16 years, patients were tetraplegic with areflexia, contractures, and scoliosis, and required assisted respiratory ventilation. Intelligence was preserved. The mild phenotype in one patient had a delayed onset (11.5 years), a moderate generalized weakness, and a slower course. Genetic testing ruled out linkage to the 5q13 and 21q22 chromosomal regions and SMN1 and SODI loci. This novel LMND variant was assigned to a locus on chromosome 1p36. (Maystadt I, Zarhrate M, Leclair-Richard D et al. A gene for an autosomal recessive lower motor neuron disease with childhood onset maps to 1p36. Neurology July (1 of 2) 2006;67:120-124). (Reprints: Dr L Viollet, Hopital Necker Enfants Malades, 149 rue de Sevres, F-75743 Paris Cedex 15, France).

COMMENT. Studies are underway to identify the gene involved in this novel variant of autosomal recessive LMND. Numerous phenotypes of childhood-onset LMND have been described, the most common being the proximal spinal muscular atrophy (SMA), linked to the SMNI gene. Kugelberg-Welander disease (SMA type III) has the same range of age at onset (after age of walking) as this novel variety, but the foot and hand paralysis, severe respiratory involvement, and lack of linkage to chromosome $5 \mathrm{q} 13$ that characterize the variant exclude the SMA III diagnosis. 